\title{
Numerical analysis of coherent many-body currents in a single atom transistor
}

\author{
A. J. Daley, ${ }^{1,2}$ S. R. Clark, ${ }^{3}$ D. Jaksch, ${ }^{3}$ and P. Zoller ${ }^{1,2}$ \\ ${ }^{1}$ Institute for Quantum Optics and Quantum Information of the Austrian Academy of Sciences, A-6020 Innsbruck, Austria \\ ${ }^{2}$ Institute for Theoretical Physics, University of Innsbruck, A-6020 Innsbruck, Austria \\ ${ }^{3}$ Clarendon Laboratory, University of Oxford, Parks Road, Oxford OX1 3PU, United Kingdom
}

(Received 29 June 2005; published 28 October 2005)

\begin{abstract}
We study the dynamics of many atoms in the recently proposed single-atom-transistor setup [A. Micheli, A. J. Daley, D. Jaksch, and P. Zoller, Phys. Rev. Lett. 93, 140408 (2004)] using recently developed numerical methods. In this setup, a localized spin-1/2 impurity is used to switch the transport of atoms in a onedimensional optical lattice: in one state the impurity is transparent to probe atoms, but in the other acts as a single-atom mirror. We calculate time-dependent currents for bosons passing the impurity atom, and find interesting many-body effects. These include substantially different transport properties for bosons in the strongly interacting (Tonks) regime when compared with fermions, and an unexpected decrease in the current when weakly interacting probe atoms are initially accelerated to a nonzero mean momentum. We also provide more insight into the application of our numerical methods to this system, and discuss open questions about the currents approached by the system on long time scales.
\end{abstract}

DOI: 10.1103/PhysRevA.72.043618

PACS number(s): 03.75.Lm, 42.50.-p, 03.67.Lx

\section{INTRODUCTION}

The recently proposed single-atom-transistor (SAT) setup [1] provides new opportunities to experimentally examine the coupling of a spin-1/2 system with bosonic and fermionic modes. Such couplings form fundamental building blocks in several areas of physics. For example, atoms passing through a cavity can allow the quantum nondemolition (QND) readout of single-photon states in quantum optics [2], and in solid state physics, such systems occur in singleelectron transistors [3], in studies of electron counting statistics [4], and in the transport of electrons past impurities such as quantum dots [5].

In the SAT setup, which was motivated by the significant experimental advances made recently with cold atoms in one dimension (1D) [6-8], a single spin-1/2 impurity atom $q$ is used to switch the transport of a gas of cold atoms in a 1D optical lattice (Fig. 1). The impurity atom, which can encode a qubit on two internal spin states, is transparent to a gas of probe atoms in one spin state (the "on" state), but acts as a single-atom mirror in the other (the "off" state), prohibiting transport via a quantum interference mechanism (Fig. 1). Observation of probe atoms that are initially situated to one side of the impurity, and which can constitute either a 1D degenerate Bose or Fermi gas, can then be used as a QND measurement [9] of the qubit state of the impurity atom $\left|\psi_{q}\right\rangle=\alpha|\uparrow\rangle+\beta|\downarrow\rangle[1]$ (see Fig. 1).

The long coherence times associated with atoms in optical lattices allow many-body effects to contribute coherently to the transport properties over longer time scales than is observed in other systems where bosonic and fermionic modes couple to a spin-1/2 system. This produces interesting physics in which the current of atoms passing the impurity, especially in a regime of weak coupling between probe atoms and the impurity, is sensitive to interactions between the probe atoms [1]. These effects could be directly observed in experiments, for example, via measurements of the density of probe atoms on each site of the impurity atom as a function of time.
In this paper we present a detailed numerical analysis of these currents, making use of recently developed numerical methods [10] to calculate the dynamics of the bosonic probe atoms by directly integrating the many-body Schrödinger equation in 1D on an adaptively truncated Hilbert space. When these currents are compared to analytical calculations of transmission coefficients for single particles passing the impurity atom and the related currents for a noninteracting 1D Fermi gas, significant interaction effects are observed, as first discussed in Ref. [1]. Here we provide additional insight into the time dependence of these currents, and what conclusions can be drawn from our numerical results on different time scales. We then calculate the initial currents for atoms at zero temperature diffusing past the impurity (where the initial mean momentum of the $1 \mathrm{D}$ gas, $\langle\hat{k}\rangle_{t=0}=0$, with $\hat{k}$ the operator corresponding to the quasimomentum in the lowest
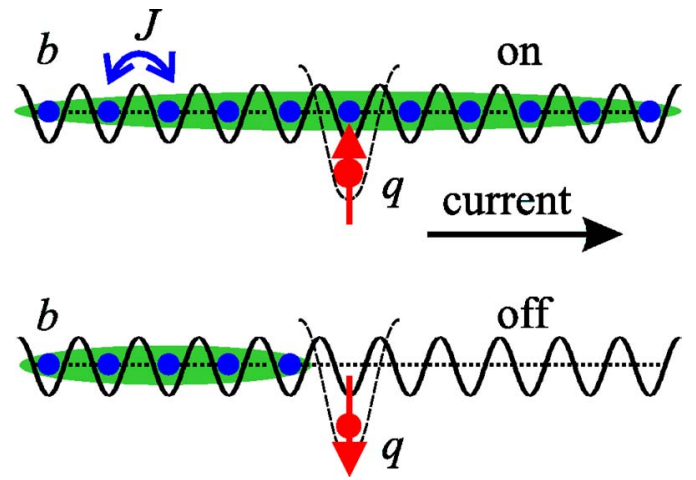

FIG. 1. (Color online) A single-atom-transistor in a 1D optical lattice. A single spin-1/2 impurity atom $q$ separately trapped at a particular lattice site is transparent to probe atoms $b$ in one state ("on"), but in the other acts as a single atom mirror ("off"). The probe atoms can either diffuse past the impurity site with mean initial momentum $\langle\hat{k}\rangle_{t=0}=0$ or can be accelerated to a finite initial momentum $\langle\hat{k}\rangle_{t=0} \neq 0$ by a kick of strength $p_{k}$. 
Bloch band and $t$ the time), and explore the effects observed for different interaction strengths of bosonic probe atoms. We then also investigate the currents for fermions and bosons when the probe atoms are initially kicked $\left(\langle\hat{k}\rangle_{t=0}\right.$ $\neq 0)$. This study is complementary to the analytical study of the SAT recently given by Micheli et al. [11].

In Sec. II we discuss the basic physics of the SAT, and give a summary of the dynamics found in [1] for single particles and noninteracting fermions. Then we present in detail the numerical techniques that we use to compute the exact time evolution of the many-body 1D system. The time dependence of the resulting currents is discussed in Sec. III, followed by a presentation of the values of the initial steadystate currents, in both the diffusive $\left(\langle\hat{k}\rangle_{t=0}=0\right)$ and kicked $\left(\langle\hat{k}\rangle_{t=0} \neq 0\right)$ regimes. The conclusions are then summarized in Sec. IV.

\section{OVERVIEW}

\section{A. The single-atom-transistor}

\section{The system}

As described in Sec. I, we consider probe atoms $b$, which are loaded into an optical lattice [12-15] with strong confinement in two dimensions, so that the atoms are restricted to move along a lattice in 1D. The probe atoms are initially situated to the left of a site containing an impurity atom $q$, which is trapped independently (by a species- or spindependent [16] potential), fixing it to a particular site while the probe atoms are free to move. In order to produce the on and off states of the SAT, we must appropriately engineer the effective spin-dependent interaction between the probe atoms and the impurity, $H_{\text {int }}=\Sigma_{\sigma} U_{\text {eff }, \sigma} \hat{b}_{0}^{\dagger} \hat{b}_{0} \hat{q}_{\sigma}^{\dagger} \hat{q}_{\sigma}$. Here, $\hat{b}_{i}^{\dagger}$ and $\hat{q}^{\dagger}$ are second-quantized creation operators for the $b$ and $q$ atoms, respectively, obeying the standard commutation (anticommutation) relations for bosons (fermions) and the site index $i$ is chosen so that the impurity is on site $i=0$. These interactions can be controlled using either a magnetic $[17,18]$ or optical [19] Feshbach resonance. For simplicity we discuss the case of an optical Feshbach resonance, depicted in Fig. 2. Here, lasers are used to drive a transition from the atomic state $\hat{b}_{0}^{\dagger} \hat{q}_{\sigma}^{\dagger}|\mathrm{vac}\rangle$ via an off-resonant excited molecular state to a bound molecular state back in the lowest electronic manifold $\hat{m}_{\sigma}^{\dagger}|\mathrm{vac}\rangle$ on the impurity site, $i=0$ (see Fig. 2). The twophoton Rabi frequency for this process is denoted $\Omega_{\sigma}$ and the Raman detuning $\Delta_{\sigma}$, and throughout this article we use units with $\hbar=1$.

\section{Single atoms}

We consider initially a single probe atom passing the impurity. If the coupling to the molecular state is far off resonance $\left(\Omega_{\sigma} \ll\left|\Delta_{\sigma}\right|\right)$, the effect of the Feshbach resonance is to modify the interaction between the $b$ and $q$ atoms in the familiar manner, with $U_{\text {eff }}=U_{q b}+\Omega_{\sigma}^{2} / \Delta_{\sigma}$. This can be used to screen the background interaction between these atoms, $U_{q b}$, so that the on state of the SAT $\left(U_{\text {eff }}=0\right)$ can be produced by choosing $\Delta_{\uparrow}=-\Omega_{\uparrow}^{2} / U_{q b}$.

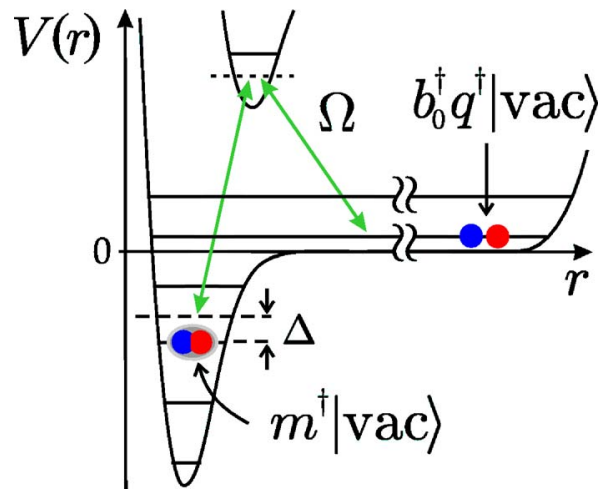

FIG. 2. (Color online) An optical Feshbach resonance for a single spin channel $\left(\Omega=\Omega_{\sigma}, \Delta=\Delta_{\sigma}\right)$. One probe atom and the impurity atom, in an atomic state $\hat{b}_{0}^{\dagger} \hat{q}_{\sigma}^{\dagger}|\mathrm{vac}\rangle$ which is quantized by the trapping potential of the lattice site, are coupled by an optical Feshbach setup to a bound molecular state $m_{\sigma}^{\dagger} \mid$ vac $\rangle$ of the BornOppenheimer potential $V(r)$ (note that here the Born-Oppenheimer potential is modified by the trapping potential of the lattice site [17]). The coupling has the effective two-photon Rabi frequency $\Omega$ and detuning $\Delta$.

If the coupling is resonant $\left(\Delta_{\downarrow}=0\right)$, then the physical mechanism is different, and the passage of a probe atom $b$ past the impurity is blocked by quantum interference. The mixing of the unbound atomic state and the molecular state on the impurity site produces two dressed states

$$
\frac{1}{\sqrt{2}}\left(\hat{b}_{0}^{\dagger} \hat{q}_{\downarrow}^{\dagger}|\mathrm{vac}\rangle \pm m_{\downarrow}^{\dagger}|\mathrm{vac}\rangle\right)
$$

with energies

$$
\varepsilon_{ \pm}=\frac{U_{q b}}{2} \pm\left(\frac{U_{q b}^{2}}{4}+\Omega_{\downarrow}^{2}\right)^{1 / 2}
$$

The two resulting paths for a particle of energy $\varepsilon$ then destructively interfere so that when $\Omega_{\downarrow} \gg J$, where $J$ is the normal tunneling amplitude between neighboring lattice sites, and $U_{q b}=0$, the effective tunneling amplitude past the impurity (see Fig. 3) is

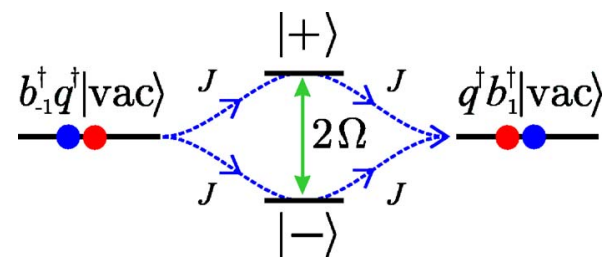

FIG. 3. (Color online) The sequence as (left) a probe atom approaches the impurity site and is located on site $i=-1$, (center) the probe atom is on the impurity site $i=0$, and (right) the probe atom has tunneled past the impurity and is located on site $i=1$. Quantum interference occurs in this process because the two dressed states on the impurity site, $| \pm\rangle=\left(\hat{b}_{0}^{\dagger} \hat{q}_{\sigma}^{\dagger}|\mathrm{vac}\rangle \pm m_{\sigma}^{\dagger}|\mathrm{vac}\rangle\right) / \sqrt{2}$, gives rise to two separate paths with equal and opposite amplitudes. 


$$
J_{\text {eff }}=\left(-\frac{J^{2}}{\varepsilon+\Omega_{\downarrow}}-\frac{J^{2}}{\varepsilon-\Omega_{\downarrow}}\right) \rightarrow 0 .
$$

This is reminiscent of the interference effect which underlies electromagnetically induced transparency [20], and corresponds to the effective interaction $U_{\text {eff }} \rightarrow \infty$ required for the off state of the SAT.

In Refs. [1,11], the Lippmann-Schwinger equation is solved exactly for scattering from the impurity of an atom $b$ with incident momentum $k>0$ in the lowest Bloch band, where the energy of the particle $\varepsilon(k)=-2 J \cos (k a)$, with $a$ the lattice spacing. The resulting transmission probabilities $T(p)$ are in the form of Fano profiles [21]. For $\Omega_{\sigma} \sim J$ these have a minimum corresponding to complete reflection for $\varepsilon(k)=-\Delta_{\sigma}$ and complete transmission for $\varepsilon(k)=-\Delta_{\sigma}$ $-\Omega_{\sigma}^{2} / U_{q b}$. For $\Omega_{\sigma}>4 J$, the transmission coefficients are approximately independent of $k$, and so complete transparency of the impurity atom is obtained for $\Delta_{\sigma}=-\Omega_{\sigma}^{2} / U_{q b}$ and complete blocking of the incident atoms for $\Delta=0$.

\section{Many atoms}

The treatment of this system for many atoms is similar to the single-atom case, but the motion of the probe atoms in the lattice, except on the impurity site, is governed by a (Bose-)Hubbard Hamiltonian [13]. As the two spin channels for the impurity atom $q$ can be treated independently, we will consider only a single spin channel $q_{\sigma}$, and drop the subscript in the notation throughout the remainder of the article [1]. The Hamiltonian for the system is then given (with $\hbar \equiv 1$ ) by $\hat{H}=\hat{H}_{b}+\hat{H}_{0}$, with

$$
\begin{gathered}
\hat{H}_{b}=-J \sum_{\langle i j\rangle} \hat{b}_{i}^{\dagger} \hat{b}_{j}+\frac{1}{2} U_{b b} \sum_{j} \hat{b}_{j}^{\dagger} \hat{b}_{j}\left(\hat{b}_{j}^{\dagger} \hat{b}_{j}-1\right), \\
\hat{H}_{0}=\Omega\left(\hat{m}^{\dagger} \hat{q} \hat{b}_{0}+\text { H.c. }\right)-\Delta \hat{m}^{\dagger} \hat{m}+U_{q b} \hat{b}_{0}^{\dagger} \hat{q}^{\dagger} \hat{q} \hat{b}_{0}+U_{b m} \hat{b}_{0}^{\dagger} \hat{m}^{\dagger} \hat{m} \hat{b}_{0} .
\end{gathered}
$$

Here, $H_{b}$ gives a Hubbard Hamiltonian for the $b$ atoms with tunneling matrix elements $J$, and collisional interactions $U_{b b}$. For fermions, $U_{b b}=0$, whereas for bosons $U_{b b}$ $=4 \pi \hbar^{2} a_{b b} \int d^{3} \mathbf{x}\left|w_{j}(\mathbf{x})\right|^{4} / m_{b}$, with $w_{j}(\mathbf{x})$ the Wannier function on site $j$, and $a_{b b}$ and $m_{b}$ the scattering length and mass of $b$ atoms, respectively. $H_{0}$ describes the dynamics in the presence of the impurity on site 0 , where atoms $b$ and $q$ are converted to a molecular state with effective Rabi frequency $\Omega$ and detuning $\Delta$, and the final two terms describe background interactions $U_{\alpha \beta}$ for two particles $\alpha, \beta \in\{q, b, m\}$, which are typically weak and will be neglected in our treatment. This single-band model is valid in the limit for $U_{\alpha \beta}, J, \Omega, \Delta \ll \omega$, where $\omega$ is the energy separation between Bloch bands, an inequality which is satisfied in current experiments. The robustness of the SAT with respect to loss processes is discussed in [1].

In the rest of this paper, we will study the current of atoms past the impurity site that develops as a function of time, and how this current depends on the interaction between probe atoms and on interactions between the probe atoms and the impurity.

\section{B. Atomic currents through the SAT}

To analyze the case of many atoms passing the impurity site, we consider the probe atoms $b$ to be prepared initially to the left of the impurity, in a ground state corresponding to a 1D box potential. The current of atoms passing the impurity is $I(t)=d \hat{N}_{R} / d t$, where $N_{R}$ is the mean number of atoms to the right of the impurity, $N_{R}=\left\langle\sum_{j>0} \hat{b}_{j}^{\dagger} \hat{b}_{j}\right\rangle$. For a sufficiently large number of atoms in the initial cloud, this current is generally found to rapidly settle into an initial steady-state current $I_{\mathrm{SS}}$ on relatively short time scales $(t J \sim 1)$ (see Sec. III A for further discussion of steady-state currents for bosons).

For a noninteracting Fermi gas at zero temperature, the currents can be calculated exactly when $U_{b m}=U_{q b}$, as the equations of motion are linear. Scattering from the impurity occurs independently for each particle in the initial Fermi sea, and after a short transient period of the order of the inverse tunneling rate $1 / \mathrm{J}$, a steady-state current $I_{\mathrm{SS}}$ is established. This can be calculated either by integrating the singleparticle transmission probabilities $[1,11]$ or by direct numerical integration of the Heisenberg equations.

For a noninteracting and very dilute Bose gas, the situation will be identical to considering a single particle. However, for higher densities, many-boson effects become important, and additionally for nonzero interactions the situation becomes even more complicated. In the limit $U_{b b} / J \rightarrow \infty$ in 1D (the Tonks gas regime) it is usually possible to replace the bosonic operators $\hat{b}_{i}, \hat{b}_{i}^{\dagger}$ by fermionic operators $\hat{f}_{i}, \hat{f}_{i}^{\dagger}$ using a Jordan-Wigner transformation [22]. However, in this case the resulting Hamiltonian

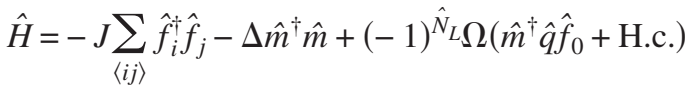

$$
\begin{aligned}
& +U_{q b} \hat{f}_{0}^{\dagger} \hat{q}^{\dagger} \hat{q} \hat{f}_{0}+U_{b m} \hat{f}_{0}^{\dagger} \hat{m}^{\dagger} \hat{m} \hat{f}_{0}
\end{aligned}
$$

contains a nonlinear phase factor resulting from the coupling on the impurity site, $(-1)^{\hat{N}_{L}}$, where $\hat{N}_{L}=\Sigma_{j<0} \hat{f}_{j}^{\dagger} \hat{f}_{j}$ is the operator for the number of atoms to the left of the impurity site. For $\Omega=0$, the boson currents are exactly the same as the currents for noninteracting fermions as $\left\langle\hat{b}_{i}^{\dagger} \hat{b}_{i}\right\rangle=\left\langle\hat{f}_{i}^{\dagger} \hat{f}_{i}\right\rangle$. For finite $\Omega$ it is not clear what role the phase factor will play in determining the system dynamics, although for sufficiently large $\Omega \gg J$ we again expect very little current to pass the impurity.

Thus, for the intermediate regime $\Omega \sim J$, and for the case of finite interaction strength $U / J$ there are no known analytical solutions for the currents. For this reason, we specifically study these regimes in this paper, using near-exact numerical methods.

\section{Time-dependent numerical algorithm for 1D many-body systems}

The algorithm that we use to compute the time evolution of our many-body system for bosonic probe atoms was originally proposed by Vidal [10]. This method allows near-exact integration of the many-body Schrödinger equation in 1D by an adaptive decimation of the Hilbert space, provided that 
the Hamiltonian couples nearest-neighbor sites only and that the resulting states are only "slightly entangled" (this will be explained in more detail below). Recently both this algorithm [23] and similar methods proposed by Verstrate and Cirac [24] have been generalized to the treatment of master equations for dissipative systems and systems at finite temperature, and progress has been made applying the latter method to 2D systems [25].

In $1 \mathrm{D}$, these methods rely on a decomposition of the many-body wave function into a matrix product representation of the type used in density matrix renormalization group (DMRG) calculations [26], which had previously been widely applied to find the ground state in 1D systems. The time-dependent algorithms have now been incorporated within DMRG codes [27], and have also been used to study the coherent dynamics of a variety of systems [28,29]. In our case, we write the coefficients of the wave function expanded in terms of local Hilbert spaces of dimension $S$,

$$
|\Psi\rangle=\sum_{i_{1}, i_{2}, \ldots, i_{M}=1}^{S} c_{i_{1}, i_{2}, \ldots, i_{M}}\left|i_{1}\right\rangle \otimes\left|i_{2}\right\rangle \otimes \cdots \otimes\left|i_{M}\right\rangle,
$$

as a product of tensors

$$
c_{i_{1}, i_{2}, \ldots, i_{M}}=\sum_{\alpha_{1}, \ldots, \alpha_{M-1}}^{\chi} \Gamma_{\alpha_{1}}^{[1] i_{1}} \lambda_{\alpha_{1}}^{[1]} \Gamma_{\alpha_{1} \alpha_{2}}^{[2] i_{2}} \lambda_{\alpha_{2}}^{[2]} \Gamma_{\alpha_{3} \alpha_{4}}^{[2] i_{2}} \cdots \Gamma_{\alpha_{M-1}}^{[M] i_{M}}
$$

These are chosen so that the tensor $\lambda_{\alpha}^{[l]}$ specifies the coefficients of the Schmidt decomposition [30] for the bipartite splitting of the system at site $l$,

$$
|\psi\rangle=\sum_{\alpha=1}^{\chi_{l}} \lambda_{\alpha}^{[l]}\left|\phi_{\alpha}^{[1, \ldots, l]}\right\rangle\left|\phi_{\alpha}^{[l+1, \ldots, M]}\right\rangle
$$

where $\chi_{l}$ is the Schmidt rank, and the sum over remaining tensors specifies the Schmidt eigenstates $\left|\phi_{\alpha}^{[1, \ldots, l]}\right\rangle$ and $\left|\phi_{\alpha}^{[l+1, \ldots, M]}\right\rangle$. The key to the method is twofold. First, for many states corresponding to a low energy in 1D systems we find that the Schmidt coefficients $\lambda_{\alpha}^{[l]}$, ordered in decreasing magnitude, decay rapidly as a function of their index $\alpha$ (this is what we mean by the state being "slightly entangled") [10]. Thus the representation can be truncated at relatively small $\chi$ and still provide an inner product of almost unity with the exact state of the system $|\Psi\rangle$. Second, when an operator acts on the local Hilbert state of two neighboring sites, the representation can be efficiently updated by changing the $\Gamma$ tensors corresponding to those two sites, in a number of operations that scales as $\chi^{3} S^{3}$ for sufficiently large $\chi$ [10]. Thus, we represent the state on a systematically truncated Hilbert space, which changes adaptively as we perform operations on the state.

In order to simulate the time evolution of a state, we perform a Suzuki-Trotter decomposition [31] of the time evolution operator $\exp (-i \hat{H} t)$, which is applied to each pair of sites individually in small time steps $\delta t$. Initial states can also be found using an imaginary time evolution, i.e., the repeated application of the operator $\exp (-\hat{H} \delta t)$, together with renormalization of the state.

In this paper, results are produced not only using the original algorithm as presented in [10], but also using an optimized version in which the Schmidt eigenstates are forced to correspond to fixed numbers of particles. This allows us to make use of the total number conservation in the Hamiltonian to substantially increase the speed of the code, and also improve the scaling with $\chi$ and $S$. With this number conserving code we are able to compute results with much higher values of $\chi$; however, we also find that for insufficiently large $\chi$, the results from this code become rapidly unphysical, in contrast to the original code (see Sec. III A).

In implementations of this method we vary the value of $\chi$ to check that the point at which the representation is being truncated does not affect the final results. A useful indicator for convergence of the method is the sum of the Schmidt coefficients discarded in each time step, although in practice the convergence of calculated quantities (such as the singleparticle density matrix $\left.\left\langle\hat{b}_{i}^{\dagger} \hat{b}_{j}\right\rangle\right)$ are normally used. This is also discussed further in Sec. III A.

For bosons on an optical lattice we must also choose the dimension $S$ of the local Hilbert space, which corresponds to one more than the maximum number of atoms allowed on one lattice site. For simulation of the SAT, we allow a variable dimension of the local Hilbert space $S_{l}$, as we must consider the state of the molecule on the impurity site in addition to the probe atoms. Allowing such a variable dimension dramatically reduces the simulation time, which scales as $\chi^{3} \Sigma_{l} S_{l}^{3}$ when $\chi \gg S$, and scales proportional to $S^{4}$ when $\chi$ is small. For a Bose gas with finite $U / J$ we usually take $S_{l}$ $=6$ away from the impurity site, and $S_{0}=12$ on the impurity site, whereas simulations of a Tonks gas can be performed with $S_{l}=2$ away from the impurity site and $S_{0}=4$ on the impurity site.

\section{NUMERICAL RESULTS}

In Sec. III A we discuss the time dependence of the current for bosons and the applicability of our numerical methods in different regimes. We establish the existence of an initial steady-state current $I_{\mathrm{SS}}$ that appears on a time scale $t J \sim 1$, and discuss the observation of a second steady-state current $I_{0}$, observed in some cases on a time scale $t J \sim 10$. In Secs. III B and III C we then present our numerical results for $I_{\mathrm{SS}}$ for the case where the initial cloud diffuses past the impurity site and the case where the initial cloud is kicked, respectively.

We are primarily interested in the behavior of the current through the SAT when it is used in the off state, i.e., we choose $\Delta=0$. To enhance clarity of the results, we also choose $U_{b q}=U_{b m}=0$.

In each case, we considered an initial cloud of between $N=1$ and 30 atoms, confined on $M=30$ lattice sites situated immediately to the left of the impurity site. The initial state used corresponds to the ground state $\left|\phi_{0}\right\rangle$ of a Bose-Hubbard model with a box trap, and was computed by preparing the 


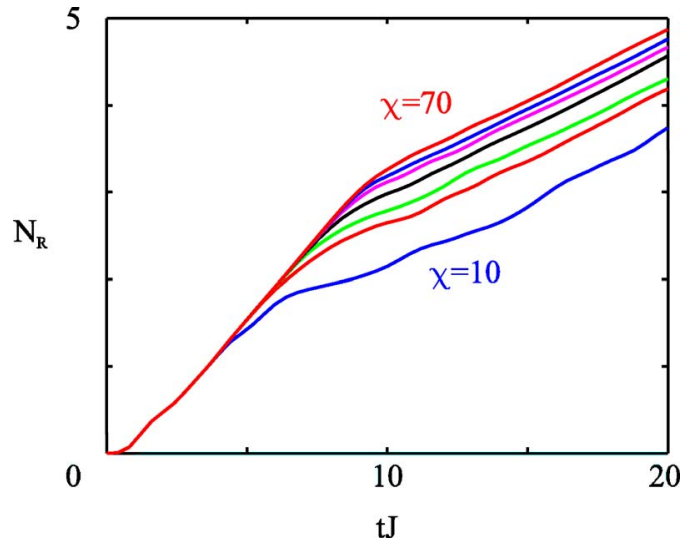

FIG. 4. (Color online) The number of atoms to the right of the impurity site $N_{R}$ as a function of dimensionless time $t J$ for a Bose gas in the Tonks limit $\left(U_{b b} / J \rightarrow \infty\right)$ with $\Omega / J=1, n=N / M=1$, and varying number of states retained in the method, $\chi$ $=10,20,30,40,50,60,70$ (lines from bottom to top). These results are from the original simulation method.

ground state on the occupied half of the system using imaginary time evolution on $M=30$ lattice sites, and constructing the remaining (empty) part of the state decomposition by hand with initial $\chi_{l}=1$ for $l \geqslant 30$.

Our total grid for the time evolution consisted of 61 lattice sites, with the 30 rightmost sites initially unoccupied, and the results we present are, except for very small systems, independent of the size of the initial cloud and of the grid size. Fermionic results are derived from exact integration of the Heisenberg equations of motion, whereas bosonic results are near-exact simulations as described in Sec. II C.

\section{A. Time dependence of the current for bosonic probe atoms}

The mean number of probe atoms on the right of the impurity $N_{R}$ is plotted as a function of time $t$ in Fig. 4 for a Tonks gas $(U / J \rightarrow \infty)$ with $\Omega / J=1$ and initial state of density $n=N / M=1$. These results were calculated with the original simulation algorithm, and it is clear from the figure that the current settles into an initial steady-state value $I_{\mathrm{SS}}$ on the time scale $t J \sim 1$. However, as is typical for bosonic probe atoms with $n>0.5$, there exists a knee in the curve at a time $t_{\text {knee }}(\chi)$, leading to a new and final steady-state current, which we will denote $I_{0}$. The time $t_{\text {knee }}(\chi)$ depends on the initial density $n$ and coupling $\Omega$, and as can be seen from this figure, we require a high value of $\chi$ to find the exact time. For $n=1, \Omega / J=1, t_{\text {knee }}(\chi)$ appears to converge to a value between $t J=9$ and 12 as $\chi$ is increased. It is clear that a significant level of correlation, or entanglement between the left- and right-hand sides of the system (in the sense of the number of significant Schmidt eigenvalues for a bipartite splitting), are involved in determining the dynamics leading to the knee. However, the actual value of the steady-state current $I_{0}$ appears to converge for much lower values of $\chi$ and there is essentially no change in this result from $\chi$ $=10$ to 70 .

The interpretation of these results is more complex when they are compared with similar results from the new, number

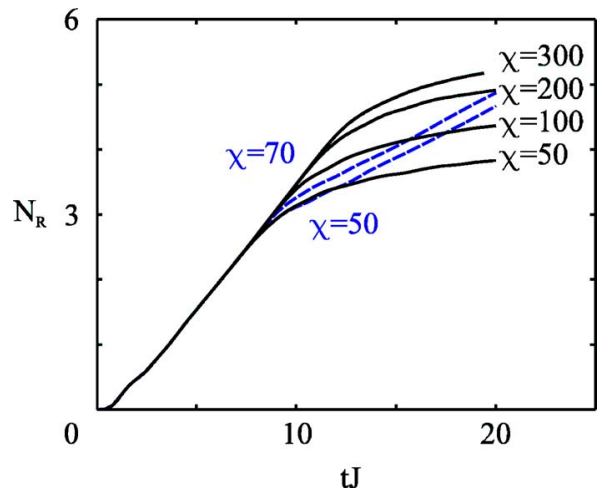

FIG. 5. (Color online) The number of atoms to the right of the impurity site $N_{R}$ as a function of dimensionless time $t J$ for a Bose gas in the Tonks limit $\left(U_{b b} / J \rightarrow \infty\right)$ with $\Omega / J=1, n=N / M=1$. This plot shows a comparison of results from the original method (dashed lines, $\chi=50,70$; cf. Fig. 4), and from the number conserving method (solid lines, $\chi=50,100,200,300$ ).

conserving version of our code. In Fig. 5 we observe that the behavior diverges at the same value of $t_{\text {knee }}(\chi)$, and even for $\chi=300$, the value of $t_{\text {knee }}(\chi)$ has only shifted a little further from where it was observed for $\chi=70$ with the original version of the code. This confirms that the dynamics on this time scale are dominated by the significant level of correlation, or entanglement between the left- and right-hand sides of the system.

In contrast to the steady-state current $I_{0}$ obtained using the original code, though, the current in the number conserving simulations rapidly approaches 0 , even for $\chi=300$. As can be seen from the dotted line in Fig. 6, this behavior occurs when the maximum sum of squares of the Schmidt coefficients being discarded in each time step, $\varepsilon_{\lambda}=\Sigma_{\beta>\chi} \lambda_{\beta}^{2}$, reaches a steady value on the order of $10^{-7}$, indicating that the simulation results from the number conserving code are probably not valid for $t>t_{\mathrm{knee}}$. Indeed, we observe the same behavior from the number conserving code with $\Omega=0$, where we know from Eq. (5) that the time-dependent current $I(t)$ is equal to that for fermions, and should not decrease in this

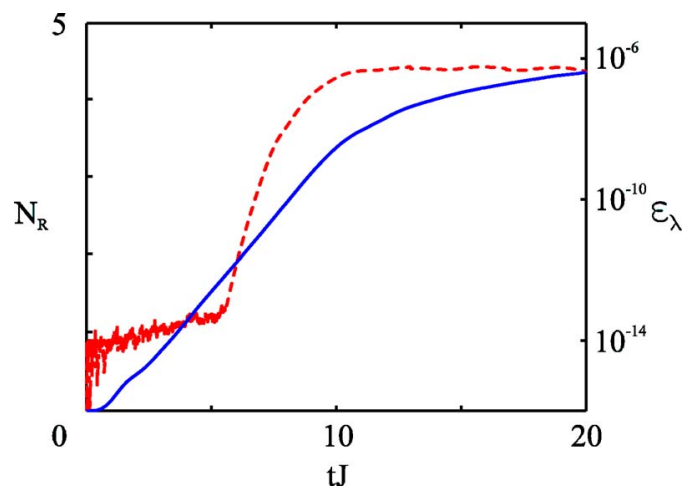

FIG. 6. (Color online) Comparison as a function of dimensionless time $t J$ of the number of atoms to the right of the impurity site $N_{R}$ (solid line), and the sum of squares of the discarded Schmidt eigenvalues $\varepsilon_{\lambda}=\Sigma_{\beta>\chi} \lambda_{\beta}^{2}$ (dashed line). These results are taken from the number conserving simulation method with $\chi=100$, for a Bose gas in the Tonks limit $\left(U_{b b} / J \rightarrow \infty\right)$ with $\Omega / J=1, n=N / M=1$. 

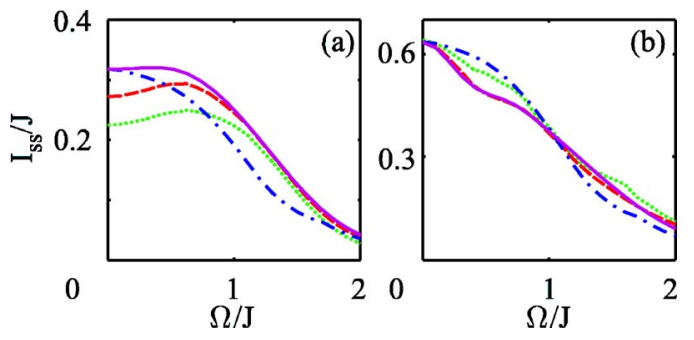

FIG. 7. (Color online) Steady-state currents through the SAT $I_{\mathrm{SS}}$ as a function of the coupling between probe atoms and the impurity $\Omega / J$. These plots show the comparison of a Bose gas with different interaction strengths $U / J=4$ (dotted line), $U / J=10$ (dashed), and $U / J \rightarrow \infty$ (solid), and a Fermi gas (dash-dotted), with $n=$ (a) $1 / 2$ and (b) 1 . In both cases, $\Delta=U_{q b}=U_{b m}=0$.

manner (see currents for fermions in Ref. [1]). Interestingly the original code, which produces the steady-state currents $I_{0}$ at finite $\Omega$, reproduces the known result at $\Omega=0$ exactly even for small values of $\chi$, with a steady-state current $I_{\mathrm{SS}}$ and no knee. This behavior for the number conserving code is thus a little surprising, especially as similar effects are not observed when time-dependent DMRG implementations with conservation of total magnetization are applied to spin chains [29]. A fuller comparison of error accumulation in the number conserving code would be an interesting topic for future investigation, but the difference between the two codes after $t_{\text {knee }}$ does not change any of the results presented in the remainder of this paper.

Our conclusions from these results are as follows.

(i) We know that up to $t_{\text {knee }}$ our simulation results are exact, as they are unchanged in the linear region with current $I_{\text {SS }}$ for $\chi=20 \rightarrow 300$. As this regime lasts at least until $t J$ $\sim 10$, these results would be observable in an experimental implementation of the SAT.

(ii) As an impractically large value of $\chi$ would be required to reproduce the results exactly on long time scales, we cannot be certain what the final behavior will be for $t>t_{\text {knee }}(\chi$ $=300$ ). This depends on clearly interesting phenomena that arise from strong correlations between the left and right sides of the impurity site, and could include settling to a final steady-state current $I_{0}$. These effects would also be observable in an experiment.

The expected final steady-state values $I_{0}$ are already discussed in Ref. [1], and so in the remainder of this paper we investigate the initial steady-state currents $I_{\mathrm{SS}}$ in various parameter regimes.

\section{B. Diffusive evolution, with initial mean momentum $\left(\langle\hat{k}\rangle_{t=0}=0\right)$}

We first consider the motion of atoms past the impurity site in the diffusive regime, where the initial state at $t=0$ is the ground state of a Bose-Hubbard model on $M=30$ lattice sites in a box trap.

\section{Dependence of the current on impurity-probe coupling $\Omega$}

In Fig. 7 we show the initial steady-state current $I_{\mathrm{SS}}$ as a function of $\Omega / J$ for fermionic probe atoms, and for bosonic probe atoms with $U_{b b} / J=4,10, \infty$ and $\Delta=0$. All of these

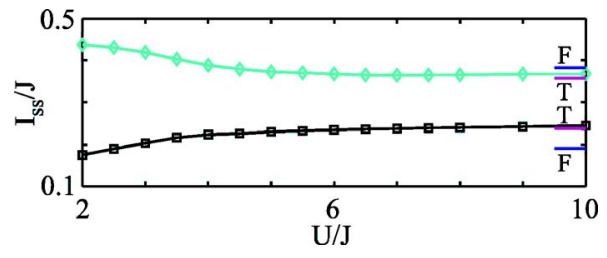

FIG. 8. (Color online) Steady-state currents through the SAT $I_{\mathrm{SS}}$ as a function of the interaction strength $U / J$ for bosonic probe atoms initially at half filling, $n=1 / 2$ (squares), and at unit filling, $n=1$ (diamonds), with $\Omega / J=1$. The equivalent results for the Tonks gas $(U / J \rightarrow \infty)$ and fermions are marked on the right-hand site of the plot. $\Delta=U_{q b}=U_{b m}=0$.

results decrease as expected with increasing $\Omega / J$, and even for a relatively small $\Omega=2 J$ the current is minimal in each case. At half filling [Fig. 7(a)], the results for the Tonks gas are identical to the Fermi results for $\Omega=0$, but become substantially different as $\Omega$ increases, with the currents in this regime greater for the bosons. At weaker interactions the currents are smaller than the Tonks result at all $\Omega$, but for $\Omega / J>1$ the currents for $U / J=4$ are larger than for a noninteracting Fermi gas. The variation in the currents for different interaction strengths of bosons appears to be due to the broader initial momentum distributions that occur at larger $U / J$. At unit filling [Fig. 7(b)], $I_{\mathrm{SS}}$ is less dependent on the interaction strength, with all of the bosonic results very close to one another, currents becoming larger than that for fermions when $\Omega / J>1$.

\section{Dependence of the current on interaction strength $U / J$}

The dependence of the initial steady-state current $I_{\mathrm{SS}}$ on the interaction strength for bosons is depicted more clearly in Fig. 8, both at unit filling, $n=1$, and half filling, $n=1 / 2$, for $\Omega=J$. At half filling the current increases with increasing $U / J$, which is due to the broader initial momentum distribution produced by the higher interaction energies. In contrast, at higher densities (here $n=1$ ), the probe atoms are blocked better by the SAT for higher interaction strengths, and $I_{\text {SS }}$ decreases. The key principle here is that bosons appear to be better blocked when they approach the impurity individually. For high densities this is achieved when large interaction strengths eliminate the higher occupancies of all lattice sites including the impurity site. For weaker interactions the bosons can swamp the transistor, with one atom being bound to the impurity, while other probe atoms tunnel onto and past the impurity site.

This effect is seen in Fig. 9, where the molecular occupation and average probe atom occupation on the impurity site are shown for $U / J=$ (a) 4 and (b) 10 . We see that as $n$ increases, the molecular occupation becomes rapidly higher for $U / J=10$ than for $U / J=4$, despite the larger occupation of probe atoms on the impurity site for $U / J=4$. This indicates that for $U / J=10$ atoms arrive individually at the impurity site, where they are coupled with the impurity atom into a molecular state, and their transport is efficiently blocked. For $U / J=4$, more than one atom enters the impurity site at once, leading to a larger average probe atom occupation on the 

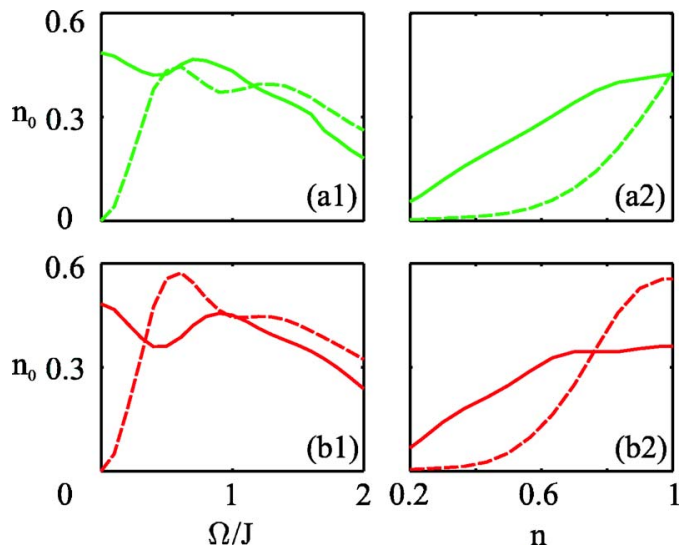

FIG. 9. (Color online) Plot showing the average steady-state occupation of the molecular state (dashed lines) and the average steady-state atomic occupation of the impurity site (solid lines) for $U / J=$ (a) 4 and (b) 10 , as a function of (a1), (b1) $\Omega / J$ with $n=1$ and (a2), (b2) $n$ with $\Omega / J=0.5$. In all cases, $\Delta=U_{q b}=U_{b m}=0$, and calculations were performed for $M=30$.

impurity site, but a comparatively small molecular occupation.

It is important to note, however, that even when $U / J=4$, the resulting currents are only slightly larger than they are for noninteracting fermions. At higher interaction strengths we then see an even stronger suppression of the steady state current for dense, strongly interacting bosons. As $\Omega$ increases, both the molecular occupation and probe atom occupation on the impurity site decrease (Fig. 9) as the probability of even a single atom tunnelling onto the impurity site becomes small. For $\Omega>2 J$ the blocking mechanism of the SAT functions extremely well even in the regime where the probe atoms are dense and weakly interacting.

\section{Dependence of the current on initial density $n$}

In Fig. 10 we show the dependence of the initial steadystate current $I_{\mathrm{SS}}$ on the initial filling factor $n$ with $\Omega / J=(\mathrm{a})$ 0.5 and (b) 1 . In both cases, the currents for bosons of different interaction strengths are very similar, with the variations following the patterns discussed in the preceding section. These results also agree well with the results for
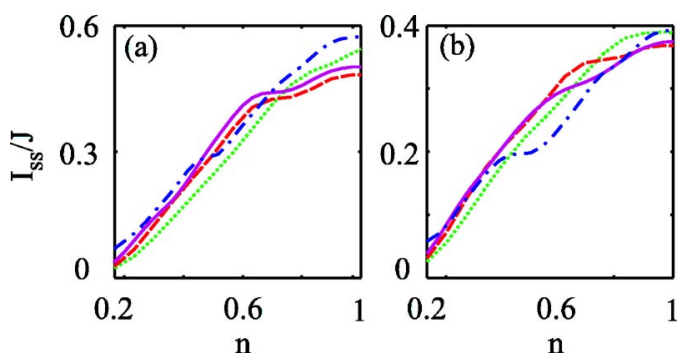

FIG. 10. (Color online) Steady-state currents through the SAT $I_{\mathrm{SS}}$ as a function of the initial density of atoms $n=N / M$. These plots show the comparison of a Bose gas with different interaction strengths $U / J=4$ (dotted line), $U / J=10$ (dashed), and $U / J \rightarrow \infty$ (solid), and a Fermi gas (dash-dotted), with $\Omega / J=$ (a) 0.5 and (b) 1 . In both cases, $\Delta=U_{q b}=U_{b m}=0$.
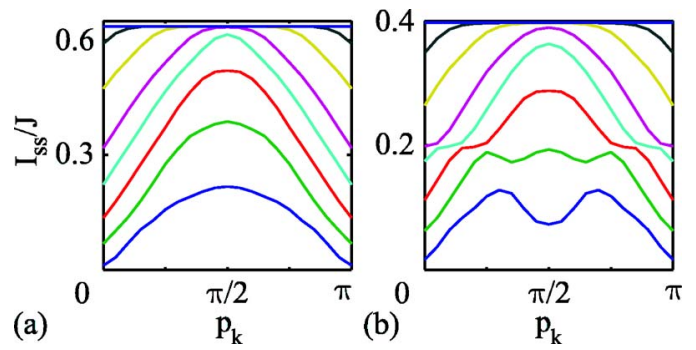

FIG. 11. (Color online) Steady-state currents $I_{\mathrm{SS}}$ through the SAT for fermions as a function of the kick parameter $p_{k}$, for (a) $\Omega=0$ and (b) $\Omega / J=1$. In each plot the lines from bottom to top sequentially correspond to filling of $N=3,6,9,12,15,20,25,30$ particles initially on $M=30$ lattice sites. Note that the scales are different for (a) and (b), and also that the results in (a) are exactly the same as those for a Tonks gas of bosons. In both cases, $\Delta$ $=U_{q b}=U_{b m}=0$.

fermions at small $n$ and for $n \sim 1$, but the plateau observed in fermionic currents near $n \sim 0.5$ does not occur in the currents for bosons. For fermions, this plateau arises from the transmission profile of the SAT as a function of incoming momentum [1], and occurs when the Fermi momentum is raised past the minimum in this transmission profile. For interacting bosons, this correspondence between the momentum distribution of the gas and the transmission profile is destroyed by many-body effects, and we see instead a smooth increase in the current. This results in the bosonic currents being substantially larger than those for fermions near half filling when $\Omega \sim 1$ [as was previously observed in Fig. 7(a)].

\section{Kicked evolution, with initial mean momentum $\left(\langle\hat{k}\rangle_{t=0} \neq 0\right)$}

In this section we consider an initial state with a nonzero initial momentum, which is obtained, e.g., by briefly tilting the lattice on a time scale much shorter than that corresponding to dynamics of atoms in the lattice. If the tilt is linear, the resulting state will be given by

$$
|\phi(t=0)\rangle=\sum_{j} \exp \left(i p_{k} j \hat{b}_{j}^{\dagger} \hat{b}_{j}\right)\left|\phi_{0}\right\rangle,
$$

where $\left|\phi_{0}\right\rangle$ is the initial many-body ground state, and the quantity $p_{k}$ is determined by the magnitude and duration of the tilt. The effect of this tilt is to translate the ground state in the periodic quasimomentum space by a momentum $p_{k}$. The final mean momentum $\langle k\rangle$ then depends on both the value $p_{k}$ and the properties of the initial momentum distribution.

\section{Dependence of the current on kick strength $p_{k}$}

In the case of fermions, the dependence of the current on $q$ for different filling factors $n=N / M$ and $\Omega$ can be clearly understood in terms of the SAT transmission profile (see [11]). In Fig. 11(a) we see the current $I_{\mathrm{SS}}$ as a function of $p_{k}$ with $\Omega=0$. The currents are each peaked at $p_{k}=\pi / 2$, where the resulting mean velocity of the probe atoms is the largest. For $N / M=1$, the whole Bloch band is filled, and the momentum distribution is not changed by the application of the kick, i.e., $\langle\hat{k}\rangle_{t=0}=0$. In Fig. 11(b) the same results are shown, 

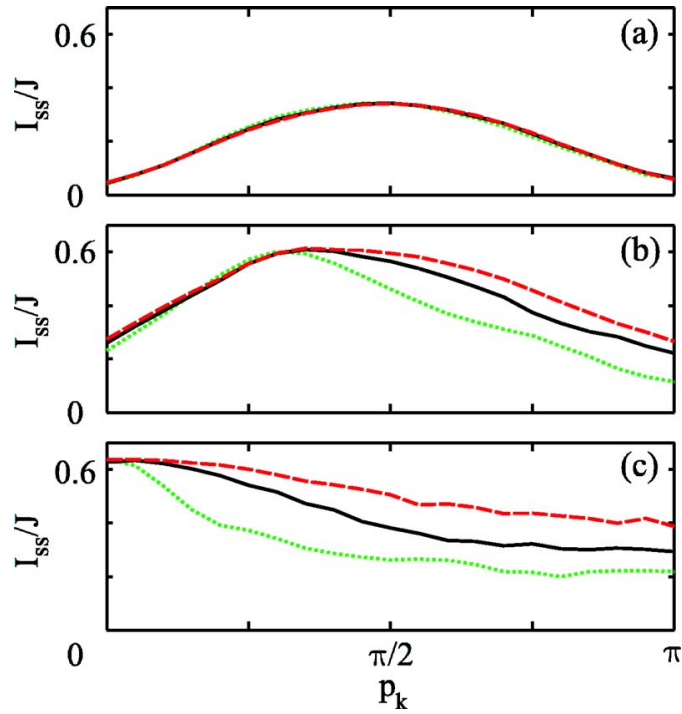

FIG. 12. (Color online) Steady-state currents with coupling to the SAT, $\Omega=0, I_{\mathrm{SS}}$ as a function of the kick parameter $p_{k}$ for varying interaction strengths, $U / J=4$ (dotted), 7 (solid), and 10 (dashed), for $N=$ (a) 5, (b) 15, and (c) 30 particles initially situated on $M=30$ lattice sites. In all cases, $\Delta=U_{q b}=U_{b m}=0$.

but with $\Omega / J=1$. Here we see that for small filling factors, a minimum appears at $p_{k}=\pi / 2$, corresponding to the minimum in the transmission profile of the SAT for this incident momentum $[1,11]$. At higher filling factors, this feature of the transmission profile for $\Omega / J=1$ is not sufficiently broad to overcome the increased current due to higher mean velocities in the initial cloud, and the peak at $p_{k}=\pi / 2$ reappears. The currents here are, of course, reduced in comparison with those for $\Omega=0$.

While for all $p_{k}$ the currents with no coupling to the impurity atom, i.e., $\Omega=0$, are the same for the Tonks gas as for fermions [Fig. 11(a)], the currents for finite interaction strengths are found to be remarkably different. In Fig. 12 these rates are plotted for $U / J=4,7,10$ for $N=5,15,30$ particles initially situated on $M=30$ sites. For the very dilute system with $N=5$ [Fig. 12(a)] we see a peak similar to that observed for fermions which is independent of the interaction strength. Here the currents are essentially those for noninteracting particles and the currents determined by the initial momentum distribution. For $N=15$ [Fig. 12(b)] we observe the surprising result that the current is peaked at a lower value than is observed for fermions or for the Tonks gas. We have observed this peak consistently for such cases of finite interaction, and note that as $U / J$ increases, the peak moves back towards $p_{k}=\pi / 2$ as the currents converge to the Tonks gas results. As $N$ is further increased, the peak continues to move left, and for $N=30$ [Fig. 12(c)] we see a monotonically decreasing current as $p_{k}$ increases. As $U / J$ increases these values tend toward the $p_{k}$-independent result observed for the Tonks gas. These results are surprising, but the trends in the behavior are clear, and they should be directly verifiable in experiments, even without the presence of the impurity atom.

For non-zero coupling to the impurity atom, the currents as a function of $p_{k}$ are shown in Fig. 13. Again we notice that
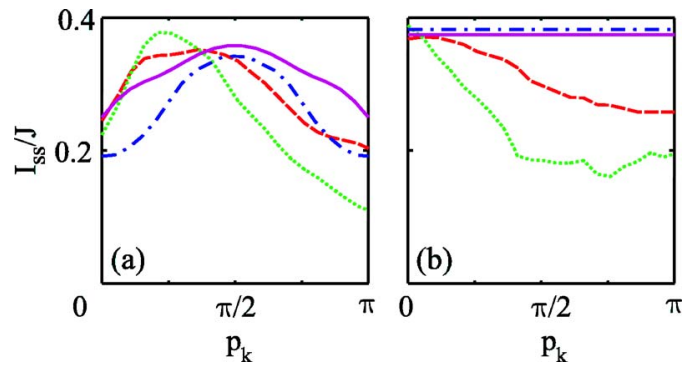

FIG. 13. (Color online) Steady-state currents through the SAT $I_{\mathrm{SS}}$ as a function of the kick parameter $p_{k}$, for $\Omega / J=1$. These plots show the comparison of a Bose gas with different interaction strengths $U / J=4$ (dotted line), $U / J=10$ (dashed), and $U / J \rightarrow \infty$ (solid), and a Fermi gas (dash-dotted), with $n=$ (a) $1 / 2$ and (b) 1 . In both cases, $\Delta=U_{q b}=U_{b m}=0$.

the current for bosons with finite interaction strength is peaked at much lower values of $p_{k}$ than the fermionic currents and that peaks of all of the bosonic currents, including the Tonks currents, as significantly larger than the fermionic currents at half filling, as was observed for diffusive results $\left(p_{k}=0\right)$. The most remarkable feature of these plots is that despite a significant reduction in the current, the basic dependence on $p_{k}$ is very similar to the $\Omega=0$ results.

\section{Dependence of the current on impurity-probe coupling $\Omega$}

The steady state current $I_{\mathrm{SS}}$ is shown in Fig. 14 as a function of $\Omega$. We observe the same strong decrease in the current due to the operation of the SAT for all of these curves, with the highest currents corresponding to the $p_{k}=\pi / 2$ curve as expected. Note that the value of $I_{\mathrm{SS}}$ is affected equally for all of the kick strengths, and the ratio in the currents for different values of $p_{k}$ is very similar for $\Omega=0$ and $\Omega / J=2$.

\section{SUMMARY}

In summary, the SAT setup provides additional experimental opportunities to study coherent transport of many atoms past a spin-1/2 impurity due to the relatively long coherence times that exist for systems of atoms in optical lattices. The resulting coherent many-body effects can be

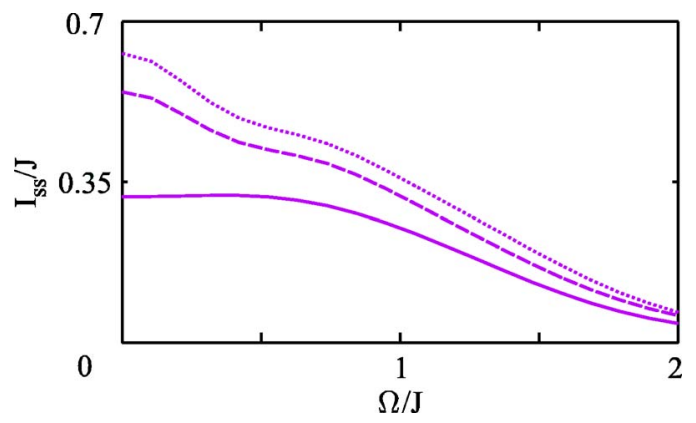

FIG. 14. (Color online) A comparison of steady-state currents through the SAT, $I_{\mathrm{SS}}$, as a function of $\Omega / J$ for $p_{k}=0$ (solid line), $\pi / 4$ (dashed), and $\pi / 2$ (dotted). Here we consider a Tonks gas $(U / J \rightarrow \infty)$ of bosonic probe atoms that is initially at half half filling, $N=15, M=30 . \Delta=U_{q b}=U_{b m}=0$. 
clearly seen in the difference between the atomic currents observed for fermions and bosons, and the nontrivial dependence of the current on interaction strength for bosons with finite interactions. Even stronger dependence on these interactions is observed when the probe atoms are initially accelerated to a nonzero momentum. The initial steady-state currents would be directly accessible quantities in the experimental implementation of the SAT, and using recently developed methods for time-dependent calculation of manybody 1D systems, we have made quantitative predictions for the corresponding currents for a wide range of system parameters. We cannot be certain about the values the currents approach at long times, although it is possible that the system will settle eventually into a regime with a different steady state current. The high values of $\chi$ needed to reproduce this behavior in our numerical calculations suggest that the currents in this regime could also be strongly sensitive to the coherence properties of the system, which would be very interesting to investigate in an experiment.

\section{ACKNOWLEDGMENTS}

The authors would like to thank A. Micheli for advice and stimulating discussions, G. Vidal for helpful discussions on the numerical methods, and A. Kantian for his contributions to producing the number conserving version of the program code. A.J.D. thanks the Clarendon Laboratory and D.J. thanks the Institute for Quantum Optics and Quantum Information of the Austrian Academy of Sciences for hospitality during the development of this work. This work was supported by EU Networks and OLAQUI. In addition, work in Innsbruck is supported by the Austrian Science Foundation and the Institute for Quantum Information, and work in Oxford is supported by EPSRC through the QIP IRC (www.qipirc.org) (Grant No. GR/S82176/01) and the Project No. EP/C51933/1.
[1] A. Micheli, A. J. Daley, D. Jaksch, and P. Zoller, Phys. Rev. Lett. 93, 140408 (2004).

[2] G. Nogues, A. Rauschenbeutel, S. Osnaghi, M. Brune, J. M. Raimond, and S. Haroche, Nature (London) 400, 239 (1999).

[3] See, for example, G. Johansson, P. Delsing, K. Bladh, D. Gunnarsson, T. Duty, A. Käck, G. Wendin, and A. Aassime, e-print cond-mat/0210163.

[4] L. S. Levitov, in Quantum Noise in Mesoscopic Physics, edited by Y. V. Nazarov (Kluwer Academic Publishers, Dordrecht, 2002).

[5] See, for example, M. A. Cazalilla and J. B. Marston, Phys. Rev. Lett. 88, 256403 (2002).

[6] T. Stöferle, H. Moritz, C. Schori, M. Köhl, and T. Esslinger, Phys. Rev. Lett. 92, 130403 (2004).

[7] B. Paredes, A. Widera, V. Murg, O. Mandel, S. Fölling, I. Cirac, G. V. Shlyapnikov, T. W. Hänsch, and I. Bloch, Nature (London) 429, 277 (2004).

[8] C. D. Fertig, K. M. O'Hara, J. H. Huckans, S. L. Rolston, W. D. Phillips, and J. V. Porto, Phys. Rev. Lett. 94, 120403 (2005)

[9] V. B. Braginsky and F. Y. Khalili, Quantum Measurement (Cambridge University Press, Cambridge, U.K., 1992).

[10] G. Vidal, Phys. Rev. Lett. 91, 147902 (2003); 93, 040502 (2004).

[11] A. Micheli and P. Zoller, e-print cond-mat/0506498.

[12] For a review on techniques for manipulating atoms in optical lattices, see D. Jaksch and P. Zoller, Ann. Phys. (N.Y.) 315, 52 (2005), and references therein.

[13] D. Jaksch, C. Bruder, J. I. Cirac, C. W. Gardiner, and P. Zoller, Phys. Rev. Lett. 81, 3108 (1998).

[14] M. Greiner, O. Mandel, T. W. Hänsch, and I. Bloch, Nature (London) 415, 39 (2002); M. Greiner, O. Mandel, T. Esslinger, T. W. Hänsch, and I. Bloch, ibid. 419, 51 (2002).

[15] M. Köhl, H. Moritz, T. Stöferle, K. Günter, and T. Esslinger, Phys. Rev. Lett. 94, 080403 (2005).

[16] O. Mandel, M. Greiner, A. Widera, T. Rom, T. W. Hänsch, and I. Bloch, Phys. Rev. Lett. 91, 010407 (2003).
[17] E. L. Bolda, E. Tiesinga, and P. S. Julienne, Phys. Rev. A 66, 013403 (2002).

[18] E. A. Donley, N. R. Claussen, S. T. Thompson, and C. E. Wieman, Nature (London) 417, 529 (2002); T. Loftus, C. A. Regal, C. Ticknor, J. L. Bohn, and D. S. Jin, Phys. Rev. Lett. 88, 173201 (2002).

[19] M. Theis, G. Thalhammer, K. Winkler, M. Hellwig, G. Ruff, R. Grimm, and J. H. Denschlag, Phys. Rev. Lett. 93, 123001 (2004).

[20] M. Lukin, Rev. Mod. Phys. 75, 457 (2003).

[21] See Fig. 3(a) in Ref. [1].

[22] See, for example, S. Sachdev, Quantum Phase Transitions (Cambridge University Press, Cambridge, U.K., 1999).

[23] M. Zwolak and G. Vidal, Phys. Rev. Lett. 93, 207205 (2004).

[24] F. Verstraete, J. J. Garcia-Ripoll, and J. I. Cirac, Phys. Rev. Lett. 93, 207204 (2004).

[25] F. Verstraete and J. I. Cirac, e-print cond-mat/0407066.

[26] S. R. White, Phys. Rev. Lett. 69, 2863 (1992); Phys. Rev. B 48, 10345 (1993); U. Schollwöck, Rev. Mod. Phys. 77, 259 (2005)

[27] A. J. Daley, C. Kollath, U. Schollwöck, and G. Vidal, J. Stat. Mech.: Theory Exp., P04005 (2004); S. R. White and A. E. Feiguin, Phys. Rev. Lett. 93, 076401 (2004).

[28] S. R. Clark, and D. Jaksch, Phys. Rev. A 70, 043612 (2004); S. R. Clark, C. Moura Alves, and D. Jaksch, New J. Phys. 7, 124 (2005); C. Kollath, U. Schollwöck, and W. Zwerger, e-print cond-mat/0504299; C. Kollath, U. Schollwöck, J. von Delft, and W. Zwerger, Phys. Rev. A 71, 053606 (2005); S. Trebst, U. Schollwöck, M. Troyer, and P. Zoller, e-print condmat/0506809.

[29] D. Gobert, C. Kollath, U. Schollwöck, and G. Schütz, Phys. Rev. E 71, 036102 (2005).

[30] M. A. Nielsen and I. L. Chuang, Quantum Computation and Quantum Communication (Cambridge University Press, Cambridge, U.K., 2000).

[31] M. Suzuki, Phys. Lett. A 146, 6 (1990); 32, 2 (1991). 